\title{
Dedicated Workforce Required to Support Large-Scale Practice Improvement
}

\author{
Shannon M. Sweeney, PhD, MPH, Jennifer R. Hemler, PhD, Andrea N. Baron, MPH, \\ Tanisha T. Woodson, PhD, MPH, Sarah S. Ono, PhD, Leab Gordon, MPH, \\ Benjamin F. Crabtree, PhD, and Deborah J. Cohen, PhD
}

Background: Facilitation is an effective approach for helping practices implement sustainable evidence-based practice improvements. Few studies examine the facilitation infrastructure and support needed for large-scale dissemination and implementation initiatives.

Metbods: The Agency for Health care Research and Quality funded 7 Cooperatives, each of which worked with over 200 primary care practices to rapidly disseminate and implement improvements in cardiovascular preventive care. The intervention target was to improve primary care practice capacity for quality initiative and the ABCS of cardiovascular disease prevention: aspirin in high-risk individuals, blood pressure control, cholesterol management, and smoking cessation. We identified the organizational elements and infrastructures Cooperatives used to support facilitators by reviewing facilitator logs, online diary data, semistructured interviews with facilitators, and fieldnotes from facilitator observations. We analyzed these data using a coding and sorting process.

Results: Each Cooperative partnered with 2 to 16 organizations, piecing together 16 to 35 facilitators, often from other quality improvement projects. Quality assurance strategies included establishing initial and ongoing training, processes to support facilitators, and monitoring to assure consistency and quality. Cooperatives developed facilitator toolkits, implemented initiative-specific training, and developed processes for peer-to-peer learning and support.

Conclusions: Supporting a large-scale facilitation workforce requires creating an infrastructure, including initial training, and ongoing support and monitoring, often borrowing from other ongoing initiatives. Facilitation that recognizes the need to support the vital integrating functions of primary care might be more efficient and effective than this fragmented approach to quality improvement. (J Am Board Fam Med 2020;33:230-239.)

Keywords: Delivery of Health Care, Evidence-Based Practice, Preventive Health Services, Primary Health Care, Qualitative Research, Quality Improvement

\section{Introduction}

Practice facilitation is an implementation strategy that has been used to help primary care practices

This article was externally peer reviewed.

Submitted 24 July 2019; revised 7 November 2019; accepted 19 November 2019.

From the Department of Family Medicine, Oregon Health \& Science University, Portland, OR (SMS, ANB, SSO, LG, DJG); Department of Family Medicine and Community Health, Research Division, Robert Wood Johnson Medical School, Rutgers University, New Brunswick, NJ (JRH, BFC); NPC Research, Portland, OR (TTW); Center to Improve Veteran Involvement in Care, VA Portland Health Care System, Portland, OR(SSO).

Funding: This work was supported by the Agency for Healthcare Research and Quality (Grant No. R01HS023940-01).

Conflicts of interest: The authors declare no potential conflicts of interest with respect to the research, authorship, and/or publication of this article. make sustainable evidence-based improvements and build capacity for continuous improvement. ${ }^{1}$ This process involves having an external facilitator or coach, trained in quality improvement (QI) methods, work with clinicians and practice staff to identify and meet improvement goals. ${ }^{2,3}$ Facilitators help practices build motivation, skills, and capacity for improvement; ${ }^{4,5}$ they provide tools, strategies, and clinical and patient educational materials for practices to use in change processes, ${ }^{6,7}$ and they assess improvement activities through audit and performance feedback. ${ }^{8-11}$ Facilitation has been

Corresponding author: Shannon M. Sweeney, $\mathrm{PhD}, \mathrm{MPH}$, 3181 Sam Jackson Park Road, Portland, OR 97239 (Email: sweenesh@ohsu.edu). 
used to implement innovative care delivery models and a variety of quality improvements. ${ }^{12}$ A systematic review of facilitated interventions found that clinicians are nearly 3 times more likely to adopt evidenced-based guidelines when supported by a facilitator. $^{13}$

While facilitation is an effective QI strategy, ${ }^{4,12-14}$ more information is needed on the infrastructure to support a successful facilitator workforce. To date, knowledge about facilitation emerges from smallscale studies in which a small number of organizations (eg, practice-based research network, academic health center) use facilitators to assist practices with QI. ${ }^{2,15}$ Recently, however, federal agencies have recognized the value of facilitation in large-scale initiatives like the federal regional extension center (REC) program, established to support practices' adoption and use of electronic health records (EHRs). While it is recognized that infrastructure is needed to ensure quality when facilitators are deployed on a large scale, little is known about what type of infrastructure and specific support is needed. ${ }^{14}$

EvidenceNOW, funded by the Agency for Health care Research and Quality (AHRQ), is an example of federal commitment to a large, facilitated practice change initiative. ${ }^{16}$ As the national evaluation for EvidenceNOW, called Evaluating System Change to Advance Learning And Take Evidence to Scale (ESCALATES), we conducted an independent, cross-Cooperative evaluation ${ }^{17}$ and had the unique opportunity to examine how 7 different grantees, called Cooperatives, developed infrastructure to support large-scale facilitation. Here, we describe the history of the Cooperatives, their partners and regional facilitation reach, the creation of the EvidenceNOW facilitation workforce, and the elements Cooperatives either leveraged or developed for scaling facilitation to rapidly disseminate and implement evidenced-based practices and guidelines.

\section{Methods}

\section{Setting and Sample}

The setting for this study is the EvidenceNOW initiative and the sample is the 7 funded Cooperatives. Cooperatives varied in the region they covered (from a single large city to multiple states), spanned 12 geographically dispersed states, engaged over 200 small-to-medium size ( $<10$ clinician) primary care practices each, and were created by bringing regional partners together. The intervention target was to improve primary care practice capacity for $\mathrm{QI}$ and the ABCS of cardiovascular disease prevention: aspirin in high-risk individuals, blood pressure control, cholesterol management, and smoking cessation. ${ }^{18,19}$ While Cooperatives designed their own interventions, all used facilitation as a core implementation strategy, provided to every practice. ${ }^{20-23}$ Cooperatives varied in using new facilitators hired for EvidenceNOW or facilitators already engaged with practices in other ongoing QI initiatives.

\section{Data Collection}

A multidisciplinary team, with expertise in qualitative methods, as well as primary care and practice improvement, public health, sociology and anthropology, collected and analyzed qualitative data for the EvidenceNOW evaluation. At the start of the initiative, we collected Cooperatives' grant applications and launched an online diary, ${ }^{24}$ a platform where Cooperative team members interacted with our team to share implementation experiences. We conducted our first site visits (August 2015 to March 2016) with Cooperatives during the EvidenceNOW start-up phase. We observed Cooperative meetings and conducted semistructured interviews with Cooperative members to learn about each region. We discussed salient regional infrastructure with members of the organizations assembled to create the Cooperatives and how Cooperatives were engaging, hiring, and training facilitators. We conducted second site visits (July 2016 to April 2017) with Cooperatives during the EvidenceNOW implementation phase. Data collection included direct observation of facilitators working with practices and how Cooperatives supported and monitored facilitators. We also conducted semistructured interviews with facilitators, their directors/managers, and leaders from organizations that employed facilitators, which we will call "facilitation organizations." These interviews explored individuals' experiences with facilitation, including how Cooperatives built or expanded infrastructure to support this workforce. Additional documents collected included facilitator tools and training materials, and facilitators' logs from Cooperatives documenting the unique work with each practice.

\section{Data Management and Analysis}

During site visits, 1 or more researchers took notes, developing these into detailed fieldnotes describing the observed activities. We assessed fieldnotes for 
accuracy and completeness. Experienced qualitative researchers familiar with the Cooperatives conducted the interviews. Interviews were audiorecorded with participant permission, and generally lasted an hour. Interviews were professionally transcribed and reviewed for accuracy. All data were deidentified and entered into Atlas.ti ${ }^{25}$ for data management and analysis.

We collected and analyzed data in real time, first using a single, broad code to tag all data relevant to facilitation. We discussed and analyzed a sample of output as a group. From this, we identified the elements of infrastructure building as an important area and created a codebook of more granular codes and definitions. We used this codebook to tag and analyze remaining data by Cooperative. We transitioned to analyzing data independently, but with regular discussion in meetings, until reaching completion. We then compared data across Cooperatives to identify the elements of each Cooperative's approach to supporting its facilitator workforce. We summarized data into preliminary findings and shared them with a wider team for feedback, prompting additional analysis and refinement.

The Oregon Health \& Science University Institutional Review Board approved this study.

\section{Results}

Cooperatives partnered with at least 1 organization in their region to establish a facilitator workforce capable of assisting over 200 practices with capacity development and rapidly disseminating and implementing the ABCS guidelines in fewer than 15 months (see Table 1). Partnerships were established, in part, with the organizations that had the ability to recruit and retain practices. Facilitation organizations providing facilitators varied, and included organizations like RECs, Quality Innovation Networks/Organizations, practice-based research networks, Academic Health Centers, health systems, and Area Health Education Centers, or a combination thereof.

Facilitation organizations varied in their history and experience working with practices on QI initiatives, though most had prior experience with facilitation (Table 1). Some had an experienced facilitator workforce honed from prior QI efforts or grants (eg, IMPaCT). Others had experience assisting practices implement EHR-related improvements (eg, Meaningful Use). Some had a combination of such experiences. Variation in
EHR experience among facilitators led some organizations to assign an EHR expert to practices, in addition to the facilitator, while other organizations had 1 person fulfilling both roles.

Table 2 shows the variation in the number of facilitation organizations for each Cooperative, the number of facilitators Cooperatives worked with, and the number of practices each Cooperative enrolled for EvidenceNOW.

The locations of facilitation organizations and their facilitators varied considerably. Facilitators in some Cooperatives were well distributed across regions, strategically placed as members of the practices' communities. Other facilitators were located more centrally and required farther travel to reach practices. Partnering with multiple facilitation organizations helped Cooperatives bring together a larger facilitator workforce and increased a Cooperative's reach across their region.

These partnerships also increased the potential for variation among facilitators and presented challenges to assuring consistency. Below, we describe the structures and processes Cooperatives had in place at the start of EvidenceNOW or implemented during the initiative to assure facilitation consistency among a large and dispersed workforce.

\section{Preparation and Training}

Facilitators' backgrounds and previous experiences varied. Some facilitation organizations had an adequate facilitator workforce they engaged in EvidenceNOW. Other facilitation organizations needed to hire new facilitators for EvidenceNOW and either formally or informally engaged their experienced facilitators as managers or mentors to new hires. Cooperatives all identified 1 or 2 team members who functioned as director of facilitation. Directors, whether in an existing role or established during implementation of EvidenceNOW, were tasked with overseeing facilitators, which included ensuring facilitators were trained and prepared to work with practices.

The purpose of training was to create consistency in how facilitators worked with practices. Cooperatives had facilitators participate in external, certified training programs. Training programs provided a general overview of how to assist practices with the change process and were particularly helpful to orient and prepare inexperienced facilitators, providing them with an overview on the art of facilitation and exposure to common tools like 
Table 1. Qualitative Descriptions of Cooperative Partnerships in Building Facilitation Workforces

\begin{tabular}{lc}
\hline Region & Organization Background and experience providing \\
facilitation support
\end{tabular}
MacColl Center for Health Care Innovation at
Kaiser Permanente Washington Health
Research Institute

Virginia

Virginia Commonwealth University

North Carolina University of North Carolina, Chapel Hill

New York City New York University

Midwest Northwestern University
Washington and Idaho

- QIN-QIO and REC, helping practices with HIT.

- PFs previously worked with practices on EHR adoption and MU, as well as other types of QI initiatives.

Oregon

- PBRN with a history of working with practices on research and QI.

- Partnered with HIT experts to provide HIT support.

- REC for VA and holds the QIO contract for VA and MD.

- PFs experienced in working with regional primary care practices. Emphasis of previous work was on EHR optimization, through a number of federal programs.

- Provided facilitation support to all practices except one large health system that used internal PFs.

- AHEC with long history in the region; experienced in developing primary care infrastructure and working with primary care practices on QI and practice redesign initiatives.

- Became the state's REC and began getting more involved in helping practices optimize use of the EHR.

- Staffs its PF workforce through a connected infrastructure of nine regional AHEC centers throughout the state. PFs are experienced in at least one of three areas - QI, data acquisition and reporting, or systems implementation and redesign - and work together to provide full-support to practices. REC

- Long history of working with small, independent practices.

- Initially assisted practices with EHR adoption and technical assistance. Now also provide services supporting PCMH recognition.

- Many PFs had previous relationships with practices and were able to quickly engage and support practices, particularly through technical support.

Primary Care Association

- 501(c)(3) non-profit receiving funding through grants for community health centers across the state. Only worked with NYC-area FQHCs on EN.

- Previously worked with practices primarily through learning collaboratives and built their knowledge of practice facilitation and their PF workforce.

- Seven organizations across the tri-state region provided support to EN practices in the Midwest. These organizations had varying roles and experience providing facilitation before EN, and varying levels of familiarity working with each other.

- Health Center Controlled Network that had previously worked with community health centers on QI projects.

- Non-profit previously participating in QI research studies, developed the curriculum and led the PF training.

- REC that previously worked with practices on MU.

- REC that previously assisted practices with MU and QI goals.

- QIO that previously assisted practices with EHR implementation, performance improvement and PQRS measurements.

- REC that previously provided HIT consulting and QI services.

- REC and QIO that assisted practices with EHR adoption and QI. 
Table 1. Continued

\begin{tabular}{lc}
\hline Region & Organization Background and experience providing \\
facilitation support
\end{tabular}

Oklahoma University of Oklahoma

Southwest University of Colorado University of New Mexico
- PF workforce builds on previous decades of work. This work was cultivated through work on past initiatives such as IMPaCT, PCMH and CPC.

- PFs were housed at the university and at the region's QIO and REC.

- Those at the QIO/REC and had more HIT experience, and previously supported practices with MU.

- QIO/REC also provided technical assistance to practices for HIT assistance.

Colorado:

- Organizations consisted of RECs, QIN-QIOs, PBRNs, integrated health networks and health systems.

Organizations have varied experience with providing PF support. One is a well-known PF organization and has trained PFs from other organizations for over a decade. Some organizations had previous relationships with their practices, and some did not.

- Most PFs assisted practices with practice redesign and QI. PFs with fewer technical skills partnered with someone with HIT skills, while others served as both the PF and worked on HIT with the practice.

New Mexico:

- QIO previously assisted private practices with QI for Medicare and CMMI.

- Nonprofit Association previously worked with FQHCs on QI projects.

- Both organizations were contractors for New Mexico's REC. Their PFs have HIT expertise and served as both PFs and worked on HIT.

EN, EvidenceNOW; REC, regional extension center; QIN-QIO, quality improvement network-quality improvement organization; PBRN, practice-based research network; MU, meaningful use; FQHC, federally quality health centers; EHR, electronic health record; QI, quality improvement; HIT, health information technology; PCMH, primary care medical home; PF, practice facilitator; AHEC, Area Health Education Center; CMMI, Center for Medicare \& Medicaid Innovation; CPC, Comprehensive Primary Care Initiative; VA, Virginia; MD, Maryland; IMPaCT, Infrastructure for Maintaining Primary Care Transformation.

motivational interviewing. Although Cooperatives reported these programs were necessary, most found them insufficient and implemented supplemental training in how to work with practices to improve cardiovascular preventive care, increase practice capacity, and measure improvement. Cooperatives also trained facilitators to use particular tools and approaches to accomplish those goals. Facilitator training was supported by grant funds and typically included a multi-day, in-person event. Training events were led by facilitator directors/ managers or other Cooperative leads and often consisted of role-playing and didactic modules. Some Cooperatives complemented training events with webinars, videos, and virtual meetings. AHRQ held regular workshops for facilitators, which included presentations by experts on multiple topics and sharing challenges and lessons learned. Facilitation organizations with less prior experience reached out to other more experienced facilitation organizations for help, which enhanced their local training and resources.

Table 2. Facilitation Workforce and Cohort Size by Cooperative

\begin{tabular}{|c|c|c|c|c|c|c|c|c|}
\hline & Co-op 1 & Co-op 2 & Co-op 3 & Co-op 4 & Co-op 5 & Co-op 6 & Co-op 7 & Total \\
\hline Number of facilitator organizations & 9 & 7 & 2 & 2 & 2 & 16 & 2 & 40 \\
\hline Number of facilitators & 31 & 17 & 17 & 16 & 19 & 35 & 23 & 158 \\
\hline Baseline practice cohort & 245 & 226 & 315 & 209 & 251 & 211 & 263 & 1720 \\
\hline
\end{tabular}


In most Cooperatives, training included opportunities to observe an experienced facilitator in a practice. Shadowing enabled facilitators to see and hear their colleagues' work first hand, and was especially useful for less experienced facilitators.

I came on without a lot of experience doing [facilitation]. [Experienced facilitator] really kind of led the training and mentorship. I worked really closely with her, and sort of shadowed her on several of her initial site visits and follow-up visits after that... I've [also] had a lot of time of listening to the coaches talk about their work. (Facilitator Interview, Cooperative 4)

\section{Ongoing Training}

For all Cooperatives, facilitator training was an ongoing process, including "booster" trainings, and Cooperatives' training sessions were developed and modified to address emerging needs (eg, motivational interviewing or plan-do-study-act cycles). Facilitators reported these booster sessions were critical to refining and learning new skills and techniques.

\section{Toolkits}

Six Cooperatives developed and provided facilitators with a toolkit at the beginning of EvidenceNOW to help guide their work; 1 Cooperative developed 1 during the initiative. Toolkits were a way to ensure consistency and structure in facilitators' work. Table 3 highlights components of Cooperatives' toolkits, including resources on building practice capacity, improving ABCS measures, and fulfilling other Cooperative-specific goals. Cooperatives adapted toolkits as needed, often at the request of facilitators, and facilitators adapted tools to practice needs. Facilitators reported that toolkits helped them work with practices. For more details, AHRQ has developed a website compiling these toolkits. ${ }^{26}$

\section{Support Structure and Processes}

Peer-to-Peer Learning

Facilitation organizations convened facilitators at regular intervals, which varied by organization (weekly, biweekly, monthly), connecting through several modes (in-person, by phone, or Web). Cooperatives also realized the benefit of bringing together facilitators from multiple facilitation organizations for peer learning.

Peer learning focused on facilitators sharing successes and challenges and engaging in group

Table 3. Practice Facilitator Toolkit Components by Cooperative

$\begin{array}{lllllll}\text { Co-op } 1 & \text { Co-op } 2 & \text { Co-op } 3 & \text { Co-op } 4 & \text { Co-op } 5 & \text { Co-op } 6 & \text { Co-op } 7\end{array}$

\footnotetext{
Clinical Tools

Materials focused on promotion of aspirin in high-risk individuals, blood pressure control, cholesterol management, and smoking cessation (ABCS) best practices and directed explicitly at ABCS change

Organizational Tools

Materials focused on the promotion of improvement of practice capacity for change and comprehensive quality improvement of the practice

Patient Education Materials

Patient resources to help with ABCS promotion (eg, patient handouts and posters)

Facilitation Instructions or Guides

Materials on how to perform practice facilitation, including examples of QI tools (eg, diagrams, agendas, and checklists)

Assessment and Goal-setting Materials

Materials used to assess practices in order to identify opportunities for improvement (excluding dashboards)

Health Information Technology Tools

Materials generated from the EHR or other electronic databases to assist in quality improvement feedback (eg, reports, dashboards)

Practice Change Model
}

Materials and PF work are grounded in a theory of change.

\begin{tabular}{|c|c|c|c|c|c|c|}
\hline$\checkmark$ & $\checkmark$ & $\checkmark$ & $\checkmark$ & $\checkmark$ & $\checkmark$ & $\checkmark$ \\
\hline$\checkmark$ & $\checkmark$ & $\checkmark$ & $\checkmark$ & $\checkmark$ & $\checkmark$ & $\checkmark$ \\
\hline \multirow[t]{2}{*}{$\checkmark$} & $\checkmark$ & $\checkmark$ & & $\checkmark$ & $\checkmark$ & $\checkmark$ \\
\hline & $\checkmark$ & $\checkmark$ & $\checkmark$ & & $\checkmark$ & $\checkmark$ \\
\hline \multirow[t]{2}{*}{$\checkmark$} & $\checkmark$ & $\checkmark$ & $\checkmark$ & $\checkmark$ & $\checkmark$ & $\checkmark$ \\
\hline & $\checkmark$ & $\checkmark$ & $\checkmark$ & & & $\checkmark$ \\
\hline$\checkmark$ & & $\checkmark$ & $\checkmark$ & & $\checkmark$ & \\
\hline
\end{tabular}

EHR, electronic health record; PF, practice facilitator; QI, quality improvement. 
brainstorming and problem solving. Facilitators and their directors/managers, and sometimes Cooperative staff, met in small subgroups to encourage discussion:

Our coaching team has a wide variety of backgrounds and expertise, and we come together weekly to discuss different variations and barriers we may be finding in practices. Sharing information openly as a team allows us to be prepared for what we may be finding in practices. (Facilitator Diary Entry, Cooperative 5)

Facilitators described looking forward to peer-topeer support and reported that it was critical to moving challenging practices forward, continuing momentum in practices that started to make changes and making breakthroughs in difficult situations: "The system that we have with the communication and the collaboration among the coaches is very strong and I think that, I would not have gotten where I am without having the support of the other coaches" (Facilitator Interview, Cooperative 1). This, in turn, helped facilitators approach challenging situations similarly to enhance fidelity and consistency.

\section{Clinical Expert Support}

In addition to peer support, some Cooperatives provided facilitators opportunities to talk with clinical experts, typically physicians from the academic health setting, to ask questions related to the ABCS guidelines and discuss practice challenges. Cooperatives that held these "clinical office hours" did so by phone and/or e-mail, as facilitators often could not attend inperson sessions. In addition to education and advice, clinical experts sometimes provided information for facilitators to share with practices. Facilitators, particularly those without clinical backgrounds and those who worked directly with clinicians, reported that access to clinical experts was useful: "When clinics have a question about ANY aspects of the cardiac measures, [the doctor's] wisdom is invaluable!" (Facilitator Diary Entry, Cooperative 2).

\section{Use of Apps and Tools for Communication and Sharing Resources}

Cooperatives used different platforms to promote archiving and sharing materials and real-time communication among facilitators. This was of critical importance, as facilitators were commonly on the road and rarely in the same physical location with colleagues. Interactive platforms included e-mail, listservs, wiki boards, and various online apps such as Slack! and GLIP. One Cooperative had its own reference librarian to gather, catalog and share information. Sharing materials reduced the amount of time facilitators spent creating or searching for information or tools and created consistency in tool use across practices. Cooperatives that did not initially provide facilitators with a communication and information-sharing platform eventually created 1 because of facilitator need:

[Cooperative] just started a listserv, which seems like it's going to work pretty well. All of us, not having to go up the chain of command, then back down the chain of command. It's just a listserv to all of our peers to be able to just say, "What are you guys looking for on statin measures?" (Facilitator Interview, Cooperative 7)

\section{Monitoring}

Cooperatives developed tracking databases for facilitators to $\log$ their activity (frequency, duration and mode) with practices. They had different ways for facilitators to notate their work, like written descriptions of visits or dropdown activity menus. To varying degrees, Cooperative directors/managers reviewed facilitators' logs and notes to monitor their work:

We have these facilitator reports and we are using that to identify what are those challenges and barriers practices have. Is this a training opportunity that we need to bring to the rest of our facilitators? Are they missing something in the knowledge or the experience that they have and do we need to bring that to the rest of team? (Facilitator Manager Interview, Cooperative 6)

Two Cooperatives carefully observed the quantity and duration of facilitator touches to ensure that practices received the intended facilitation dose, intervening when dose was not on target. Two Cooperatives found that tracking $\log$ review, particularly of facilitator notes, helped identify facilitators who may be experiencing difficulty, and fostered remediation (eg, retraining; shadowing and feedback). Another Cooperative had supervisors shadow facilitators to observe their work and offer suggestions for improvement. Director/managers found tracking critical to keeping track of a large workforce. These databases provided insight into how facilitators worked with practices and aided in fostering consistency and fidelity. For more details, AHRQ has compiled some of these tools on their Web site. ${ }^{27-29}$ Some facilitators reported that although data entry could be time consuming, databases were helpful to tracking and planning practice visits. 


\section{Discussion}

EvidenceNOW offered a unique opportunity to identify, across 7 different regions, the infrastructure needed to support facilitation at a large scale. Because our data collection followed 7 cases and compared their experiences prospectively, we were able to identify the infrastructure Cooperatives had in place at baseline, observe what adjustments were made, when adjustments were needed, and identify critical elements to support large-scale facilitation. We considered critical elements as those structures and processes that were either observed in place at baseline or added because they were determined to be important to supporting facilitators.

Our findings also show that supporting large-scale facilitation requires an investment in partnerships to cultivate a large quantity of facilitators. This includes training, facilitator support, and monitoring quality. Many facilitation organizations are separate business entities with funding streams that support their facilitators, such as RECs and QI organizations. Indeed, many of the Cooperatives were sophisticated in piecing together diverse sources of funding to support the facilitators working with their practices. Cooperatives are good at this because they have experience piecing together disparate categorical funding for usually disease-specific QI efforts. Unfortunately, this contradicts the vital function of primary care in integrating care for whole people. It would be worthwhile to consider what might be accomplished if the federal government adequately funded AHRQ to support the practice extension and primary care quality. Moreover, we should include in this consideration if practices and practice networks were, in turn, supported to facilitate the primary care function. The primary care function is about integrating, personalizing and prioritizing care, not improving 1 disease or prevention category at a time, which risks further fragmentation of care and burnout $^{30,31}$ when these improvement efforts are uncoordinated. An understanding of the care of whole people as more than the sum of their parts will be needed for a more integrated approach to emerge.

While facilitation organizations have revenue streams to support their existing workforce, many expanded their workforces for the purposes of EvidenceNOW; when EvidenceNOW ended, some of these-particularly academic health centersneeded to rescale their facilitator workforce. This creates challenges for developing a stable and skilled facilitator workforce and a durable and sustainable infrastructure to support facilitators adequately. Culler et $\mathrm{al}^{32}$ estimate that operating a primary care facilitation program can be cost-neutral because facilitator services promote quality and may prevent costs associated with hospitalizations. Our study adds important information to this body of work by detailing the basic elements of facilitation infrastructure that must also be financially supported.

A number of limitations must be noted. First, only 2 Cooperatives provided facilitation to practices in all regions of their state. While not every Cooperative accomplished or aimed to accomplish this level of reach, the infrastructure support used by all Cooperatives was similar, suggesting this study provides a good indication of the required infrastructure regardless of reach. Second, 2 Cooperatives were able to partner primarily with 1 state-based facilitation organization to develop the majority of their workforce. While this is different from regions that needed to partner with several facilitation organizations to establish an adequate facilitator workforce, our findings show that single facilitation organizations needed the same infrastructure to support facilitators as Cooperatives with multiple facilitation organizations, suggesting our findings are transferable to a number of different organizational configurations. Third, Cooperatives able to compete for EvidenceNOW funding likely were more sophisticated than the average system or network in accessing and using facilitation for QI, and certainly more so than the average unaffiliated practice. This affects the transportability of the findings to less connected or quality-focused practices and networks of practices. Fourth, while we can report the number of facilitation organizations, the number of facilitators in EvidenceNOW by Cooperative, and the number of practices each Cooperative enrolled at baseline, we cannot report facilitator workload or panel size because many facilitators worked with practices on other (non-EvidenceNOW) QI initiatives, and these data were not shared. Fifth, although our team collected qualitative data from all 7 Cooperatives at many time points and using several modes, some facilitation organizations are less represented. Sixth, our assessment of the usefulness of the identified infrastructure is based on qualitative observation and interview data. Satisfaction, usefulness and the extent to which the identified infrastructure supports facilitator efficacy or perceived self efficacy is outside the scope of this study but is an excellent avenue for 
future researchers. Lastly, this study does not examine if the infrastructural elements we identified, when present, influenced intervention fidelity and/or were aligned with greater improvements in targeted outcomes. While we have some qualitative evidence to suggest this might be so, more research is needed to formulate and test hypotheses that can establish evidence for such relationships.

\section{Conclusion}

This study of EvidenceNOW makes apparent that supporting large-scale facilitation requires thoughtful, intentional design of infrastructure to support a diverse workforce and ensure consistency and fidelity. Few regions have facilitation organizations at this scale. To establish and sustain this capacity requires single or multiple facilitation organizations to establish an infrastructure to support this workforce. This needs to include adequate initial and ongoing training, developing structures and supervisory roles to manage and mentor facilitators, and processes and tools to align facilitator work and to monitor a workforce that, when successful, is largely in the field. While we know that facilitation is effective in supporting practices through QI, few studies describe how to take these efforts to scale. More research is needed to understand how the presence or absence of these elements may influence practice engagement and improvement, and policy efforts are needed to fund the facilitation infrastructure our study suggests is needed.

It is likely that the ability to support facilitators to provide facilitation to practices, and the focus of that facilitation, varies with the availability and focus of funding. This could lead to an unstable career path for facilitators, and widely and perhaps wildly variable support for practice improvement, particularly for small practices like those in this study. If facilitation is an important pathway to practice improvement, it may make sense to find ways to make it available consistently, strategically, and in an integrated fashion, and to support the primary care function by moving beyond facilitating narrowly-focused QI to supporting the broader integrated functions of primary care that provide value within a largely fragmented system.

\footnotetext{
To see this article online, please go to: http://jabfm.org/content/ 33/2/230.full.
}

\section{References}

1. Knox L. Report on the AHRQ 2010 Consensus Meeting on practice facilitation for primary care improvement. Rockville, MD: L.A. Net, A Project of Community Partners; 2010.

2. Nagykaldi Z, Mold JW, Aspy CB. Practice facilitators: a review of the literature. Fam Med 2005;37:581-8.

3. Taylor EF, Machta RM, Meyers DS, Genevro J, Peikes DN. Enhancing the primary care team to provide redesigned care: the roles of practice facilitators and care managers. Ann Fam Med 2013;11:80-3.

4. Nutting PA, Crabtree BF, Stewart EE, et al. Effect of facilitation on practice outcomes in the National Demonstration Project model of the patient-centered medical home. Ann Fam Med 2010;8:S33-S44; S92.

5. Taylor EF, Genevro J, Peikes D, Geonnotti K, Wang W, Meyers D. Building Quality Improvement Capacity in Primary Care: Supports and Resources. Rockville, MD: Agency for Healthcare Research and Quality. 2013. Available at: https://www.ahrq. gov/sites/default/files/publications/files/pcmhqi2. pdf.

6. Harvey G, Lynch E. Enabling continuous quality improvement in practice: the role and contribution of facilitation. Front Public Health 2017;5:27.

7. Knox L. The practice facilitation handbook: training modules for new facilitators and their trainers. Rockville, MD: Agency for Healthcare Research and Policy; 2013.

8. Geonnotti K, Taylor EF, Peikes D, et al. Engaging primary care practices in quality improvement: strategies for practice facilitators (executive summary). Princeton, NJ: Mathematica Policy Research; 2015.

9. McNamara P, Shaller D, De La Mare D, Ivers N. Confidential physician feedback reports: designing for optimal impact on performance. Rockville, MD: Agency for Healthcare Research and Quality; 2016.

10. Higgins TC, Crosson J, Peikes D, McNellis R, Genevro J, Meyers D. Using health information technology to support quality improvement in primary care. Princeton, NJ: Mathematica Policy Research; 2015.

11. Sargeant J, Lockyer J, Mann K, et al. Facilitated reflective performance feedback: developing an evidence-and theory-based model that builds relationship, explores reactions and content, and coaches for performance change (R2C2). Acad Med 2015;90:1698-706.

12. Ritchie MJ, Parker LE, Edlund CN, Kirchner JE. Using implementation facilitation to foster clinical practice quality and adherence to evidence in challenged settings: a qualitative study. BMC Health Serv Res 2017;17:294.

13. Baskerville NB, Liddy C, Hogg W. Systematic review and meta-analysis of practice facilitation within primary care settings. Ann Fam Med 2012;10:63-74. 
14. Grumbach K, Bainbridge E, Bodenheimer T. Facilitating improvement in primary care: the promise of practice coaching. Issue Brief (Commonw Fund) 2012;15:1-14.

15. Nagykaldi Z, Mold JW, Robinson A, Niebauer L, Ford A. Practice facilitators and practice-based research networks. J Am Board Fam Med 2006;19:506-10.

16. Ono SS, Crabtree BF, Hemler JR, et al. Taking innovation to scale in primary care practices: the functions of health care extension. Health Aff (Millwood) 2018;37:222-30.

17. Cohen DJ, Balasubramanian BA, Gordon L, et al. A national evaluation of a dissemination and implementation initiative to enhance primary care practice capacity and improve cardiovascular disease care: the ESCALATES study protocol. Implement Sci 2016;11:86.

18. Farley TA, Dalal MA, Mostashari F, Frieden TR. Deaths preventable in the U.S. by improvements in use of clinical preventive services. Am J Prev Med 2010;38:600-9.

19. Frieden TR, Berwick DM. The "million hearts" initiative-Preventing heart attacks and strokes. N Engl J Med 2011;365:e27.21-e27-e24.

20. Weiner BJ, Pignone MP, DuBard CA, et al. Advancing heart health in North Carolina primary care: the Heart Health NOW study protocol. Implement Sci 2015;10:160.

21. Shelley DR, Ogedegbe G, Anane S, et al. Testing the use of practice facilitation in a cluster randomized stepped-wedge design trial to improve adherence to cardiovascular disease prevention guidelines: HealthyHearts NYC. Implement Science 2016;11:88.

22. Parchman ML, Fagnan LJ, Dorr DA, et al. Study protocol for "Healthy Hearts Northwest": a $2 \times 2$ randomized factorial trial to build quality improvement capacity in primary care. Implement Sci 2016;11:138.
23. Perry CK, Damschroder LJ, Hemler JR, Woodson TT, Ono SS, Cohen DJ. Specifying and comparing implementation strategies across seven large implementation interventions: a practical application of theory. Implementation Sci 2019;14:32.

24. Cohen DJ, Leviton LC, Isaacson N, Tallia AF, Crabtree BF. Online diaries for qualitative evaluation: gaining real-time insights. Am J Eval 2006;27:163-84.

25. Atlas.ti [computer program]. Berlin, Germany.

26. EvidenceNOW Tools for Change for Practice Facilitators. Agency for Healthcare Research and Quality. Available from: https://www.ahrq.gov/ evidencenow/tools/facilitation/index.html. Accessed July 8, 2019.

27. Quality AfHRa. Documenting practice facilitators' quality improvement (QI) work. 2015. Available from: https://www.ahrq.gov/evidencenow/tools/ facilitation/qi-documentation.html. Accessed October 28, 2019.

28. Quality AfHRa. Primary care quality improvement (QI) tracking worksheet. Available from: https://www. ahrq.gov/evidencenow/tools/facilitation/qi-tracking. html. Accessed October 28, 2019.

29. Quality AfHRa. Primary care quality improvement (QI) progress tracker. Available from: https://www. ahrq.gov/evidencenow/tools/facilitation/qi-report. html. Accessed October 28, 2019.

30. Stange KC. The problem of fragmentation and the need for integrative solutions. Ann Fam Med 2009; 7:100-3

31. Edwards ST, Marino M, Balasubramanian BA, et al. Burnout among physicians, advanced practice clinicians and staff in smaller primary care practices. J Gen Intern Med 2018;33:2138-46.

32. Culler SD, Parchman ML, Lozano-Romero R, et al. Cost estimates for operating a primary care practice facilitation program. Ann Fam Med 2013;11:207-11. 